Recebido em 05/2016. Aceito para publicação em 03/2017.

\title{
LINHA DE CUIDADO AO CÂNCER DE COLO DE ÚTERO E MAMA NO LITORAL NORTE PAULISTA SOB O OLHAR DE COORDENADORES DE UNIDADES DE ATENÇÃO PRIMÁRIA EM SAÚDE
}

\author{
CLINICAL PATHWAY OF CERVICAL AND BREAST CANCER IN THE NORTH \\ COAST OF SÃO PAULO ACCORDING TO PRIMARY HEALTH CARE MANAGERS \\ Natalia Barboza Helbusto ${ }^{1}$ \\ Paula Vilhena Carnevale Vianna ${ }^{2}$
}

\begin{abstract}
Resumo: Em 2015, o Plano Regional da Rede de Oncologia da Região de Saúde 17 (RRAS17) estabeleceu parâmetros de organização e encaminhamentos dos serviços de atenção primária, secundária e terciária que compõem a rede. Esta pesquisa visou avaliar a percepção dos coordenadores das Unidades Básicas de Saúde (UBSs) acerca do funcionamento da linha de cuidado ao câncer de mama e colo de útero no Litoral Norte (LN), uma das quatro regiões de saúde da RRAS17, cujos indicadores apontam lacunas no cuidado integral a portadoras dessas patologias. A aplicação de questionário estruturado mostrou que, para os coordenadores, os serviços estão integrados e o funcionamento da linha de cuidado para essas condições é cumprido, embora pontos críticos, como a educação permanente, a supervisão técnica e a participação social devam ser aprimorados.

Palavras-chave: Câncer de mama; câncer de colo de útero; linha de cuidado; rede de Saúde.
\end{abstract}

\begin{abstract}
In 2015 the Regional Plan for Cancer Health Network of Health Region 17 (RRAS17) set standards for the organization and referral of primary, specialized and hospital services that constitute the network. The current paper aims to assess the perception of Primary Care managers concerning the performance of the care pathway for breast and cervical cancer in São Paulo North Coast, one of the four health regions of RRAS 17. The health indicators of the region suggested flaws in the integral care to those patients. The structured questionnaire showed that managers consider services to be integrated and operating properly, although critical points, such as permanent education, technical supervision and social participation should be addressed.
\end{abstract}

Keywords: Breast cancer; cervical cancer; line of care; healthcare network.

\section{INTRODUÇÂO}

A urbanização e as práticas de saúde mudaram o perfil epidemiológico de adoecimento e morte das populações, levando à redução das doenças infecciosas e ao aumento das doenças crônicas não transmissíveis. No Brasil, a incidência e morte por câncer são ascendentes, seguindo a tendência mundial. O câncer de mama é a neoplasia mais comum e de maior mortalidade entre as mulheres no país, seguida do câncer colorretal e de colo de útero. Os fatores de risco para os dois tipos de câncer diferem. Enquanto o câncer de colo de útero está associado à infecção pelo papiloma vírus humano (HPV), ao tabagismo e à baixa instrução e renda das mulheres acometidas pela doença (é o tipo de câncer mais prevalente na Região Norte do país, de piores indicadores econômicos e sociais), o câncer de mama está associado à

\footnotetext{
1 Mestranda em Planejamento Urbano e Regional/Universidade do Vale do Paraíba - UNIVAP, Brasil. E-mail: natalia.helbusto@hotmail.com.

2 Docente Programa de Pós Graduação em Planejamento Urbano e Faculdade de Ciências da Saúde/IP\&D/Universidade do Vale do Paraíba - UNIVAP, Brasil. E-mail: paulavianna@univap.br.
} 
herança familiar e às mudanças da condição social e da vida reprodutiva da mulher. $A$ incidência desse câncer é mais alta na Região Sul. A despeito das diferenças, em ambos, como em todos os tipos de câncer, o acesso aos serviços de saúde é fator determinante para o controle e prognóstico, visto que a detecção precoce e tratamento oportuno influenciam diretamente a taxa de mortalidade. (INCA, 2011). E é este o objeto deste estudo, explorar o funcionamento da rede oncológica de cuidado ao câncer feminino em uma sub-região de saúde do Estado de São Paulo.

A consolidação e integração das ações de prevenção, assistência e controle de câncer de mama e colo uterino se organizam institucional e normativamente em torno de quatro compromissos prioritários, entre eles o fortalecimento das ações para a prevenção e qualificação do diagnóstico e tratamento dos cânceres do colo do útero e da mama. (BRASIL, 2011). Como forma de garantir o compromisso, em 2011 foi instituído o Plano de Ações Estratégicas para o Enfrentamento das Doenças Crônicas não Transmissíveis. (BRASIL, 2011), e o Plano de Fortalecimento das Ações para Prevenção e Qualificação do Diagnóstico e Tratamento dos Cânceres do Colo do Útero e da Mama. (BRASIL, 2013). Este último possui eixos estruturantes e ações transversais, estratégicas para a implementação da política e do funcionamento adequado dos programas nacionais para o controle dos cânceres (mama e colo de útero). Os eixos estruturantes correspondem ao fortalecimento do Programa Nacional de Controle dos Cânceres do Colo do Útero e da Mama.

Os indicadores de monitoramento e avaliação das ações implementadas incluem a razão de citopatológico de útero e de mamografia para mulheres nas faixas etárias preconizadas. A definição das metas é obrigatória no nível municipal, estadual e federal. Para atingir as metas pactuadas, o Ministério da Saúde (MS) preconiza, nas portarias e planos citados, que os municípios organizem suas ações de saúde na forma de linha de cuidado, ou seja, integrando: a. o atendimento pelo generalista (rastreamento e diagnóstico precoce); b. o atendimento especializado (investigação pelo médico especialista e acesso a exames diagnósticos, tanto laboratoriais como de imagem e anatomopatológico); c. o tratamento de alta complexidade (cirurgia ou quimioterapia), e d. os cuidados paliativos (controle dos sintomas e alívio da dor, com suporte espiritual e apoio ao cuidador), sempre tendo como base a atenção primária. As Unidades de Saúde da Família devem ser o eixo do cuidado, acompanhando todo o processo. (BRASIL, 2005, 2006, 2011). O acesso às ações e aos serviços qualificados deve possibilitar a promoção da saúde, a prevenção do câncer, o rastreamento de lesões precursoras, o diagnóstico precoce e o tratamento adequado. Em todos os pontos da rede, o cuidado deve ser humanizado e integral. Orientação recente do Ministério da Saúde preconiza 60 dias de intervalo entre o diagnóstico e o início do tratamento por câncer no SUS. (BRASIL, 2012). A integração das ações é fundamental para atingir essa meta, pois, se a detecção precoce, realizada na atenção primária influencia diretamente o prognóstico, a articulação com a rede de atenção secundária garante a confirmação diagnóstica e o tratamento especializado. Embora a organização regional 
dos serviços e o atendimento integrado tenham documentos ordenadores desde 2006. (BRASIL, 2006), estudos mostram que a articulação entre os serviços ainda é falha, o que aumenta o intervalo de tempo para o início do tratamento. (ROSA e RADNUZ, 2013).

A Região Metropolitana do Vale do Paraíba e Litoral Norte (RMVale), no Estado de São Paulo, contexto deste estudo, apresenta índice de mortalidade por câncer acima da média estadual. A mortalidade por câncer de mama é ascendente $(14,41$ óbitos/100.000 mulheres (2011); 16,17 óbitos/100.000 mulheres (2014), em ascenção embora ainda discretamente inferior à média do Estado (16,54/100.000). O número de mamógrafos é adequado na região (segundo o parâmetro do Ministério da Saúde) e a razão de mamografia é ascendente e superior à meta pactuada pelo Ministério da Saúde no ano de 2012 ( $\geq 0,35$ ) (Tabela 1). No entanto, os equipamentos se concentram nos 12 municípios mais desenvolvidos e a cobertura é insuficiente em algumas regiões de saúde e municípios. O diagnóstico, no geral, é tardio. A taxa de mortalidade por câncer de colo de útero é similar à taxa estadual, mas a razão de papanicolaou decresceu nos últimos anos, apesar do aumento do número de Unidades Básicas de Saúde (UBS) e de equipes de saúde da família. Os municípios mais desenvolvidos apresentam maior taxa de mortalidade por câncer de mama e taxa de mortalidade estacionária, por câncer de colo de útero. (HELBUSTO et al, 2014).

O Litoral Norte, sub-região estudada, apresenta infraestrutura adequada, em parâmetros numéricos, para o funcionamento da rede: são 53 UBSs, sendo 10 delas no município de Caraguatatuba, 9 em Ilhabela, 16 em São Sebastião e 18 em Ubatuba. (DATASUS); 7 mamógrafos registrados no Cadastro Nacional de Estabelecimentos de Saúde (CNES), sendo 4 disponíveis ao SUS. O número de mamografias realizadas em 2012 (5.299) foi superior à quantidade de mamografias necessárias para atingir a meta estadual (4.000 exames, ou 0,35 exames/ mulheres entre 50 e 69 anos). Embora a razão de mamografias do Litoral Norte (0,42 em 2014; Tabela 1) esteja acima da meta estadual, houve um decréscimo (0,46 em 2012) e a mortalidade por câncer de mama está em ascensão. A taxa de mortalidade por câncer de colo de útero, por sua vez, está estacionária e a razão de exames citológicos para mulheres entre 25 e 64 anos, nos 2012 e 2014, embora abaixo da meta estadual (0,75 exames/mulheres entre 25 e 64 anos), permanece acima da razão da região $(0,46$, em comparação a 0,42 na RMVale, Tabela 1). Para a realização de mamografia de rastreamento e ultrassonografia, as mulheres do Litoral Norte são encaminhadas para o Ambulatório Médico de Especialidades (AME), de Caraguatatuba. Quando diagnosticadas, são encaminhadas para o Hospital São Francisco de Assis, no município de Jacareí, na RMVale, onde iniciam o tratamento, que pode ser complementado nos municípios de Taubaté e São José dos Campos, também na RMVale. Embora os indicadores selecionados apontem para melhor desempenho e resultados no cuidado ao câncer de mama e colo de útero, em relação à região como um todo, o decréscimo na razão de mamografias e 
citopatológico de colo de útero, na região do Litoral Norte, sugere lacunas no acesso e organização dos serviços de saúde.

Tabela 1. Indicadores demográficos e de saúde selecionados para a Região Metropolitana do Vale do Paraíba e Litoral Norte (RMVale) e da Região de Saúde do Litoral Norte, relativos ao câncer de mama e câncer de colo de útero.

\begin{tabular}{lrr}
\hline & \multicolumn{2}{c}{2014} \\
\hline & RMVale & $\begin{array}{r}\text { Litoral } \\
\text { Norte }\end{array}$ \\
\hline População total & 2.430 .381 & 310.240 \\
População feminina & 1.169 .041 & 145.750 \\
Percentual de população com cobertura de plano de saúde (\%) (2012) & 34,84 & 17,26 \\
Percentual de cobertura da estratégia saúde da família (\%) & 30,94 & 83,11 \\
Percentual de cobertura da atenção básica (\%) & 64,74 & 86,41 \\
Razão de médicos SUS por 1.000 habitantes & 0,53 & 0,53 \\
Razão de enfermeiros SUS por 1.000 habitantes & 0,66 & 0,71 \\
$\begin{array}{l}\text { Razão de mamografia (exames realizados pela metade população feminina de } \\
50 \text { a 69 anos) (meta pactuada em 2012 } 0 \text { 0,35) }\end{array}$ & 0,44 & 0,42 \\
Mortalidade por câncer de mama (número de casos) & 189 & 19 \\
Mortalidade por câncer de mama (taxa por 100.000 mulheres) & 16,17 & 13,03 \\
Razão de citológicos realizado (exames realizados por 1/3 da população & & \\
feminina de 25 a 64 anos) (meta pactuada em 2012 0,75) & 0,42 & 0,46 \\
Mortalidade por câncer de colo de útero (número de casos) & 26 & 1 \\
Mortalidade por câncer de colo de útero (taxa por 100.000 mulheres) & 2,22 & 0,69 \\
\hline$\quad$ Fonte: Datasus & &
\end{tabular}

Fonte: Datasus.

Esta pesquisa teve como objetivo analisar a percepção dos gestores de unidades básicas de saúde sobre o funcionamento da linha de cuidado ao câncer de mama e colo de útero na rede regional de atenção do Litoral Norte, tendo como pano de fundo, o cenário epidemiológico delineado pelos indicadores de pactuação compulsória.

\section{METODOLOGIA}

Trata-se de uma pesquisa exploratória quantitativa, que investiga o funcionamento em rede da linha de cuidado ao câncer ginecológico feminino, na região do Litoral Norte, segundo a avaliação de coordenadores das unidades básicas de saúde do município. Este estudo completa análise anterior da estrutura da rede. (HELBUSTO et al, 2014). A escolha da linha de cuidado para o câncer ginecológico feminino se deu pela carga de doença e, igualmente, pela reconhecida legitimidade do cuidado dirigido às mulheres nas Unidades de Atenção Primária. A saúde da mulher foi uma das primeiras áreas programáticas a orientar ações preventivas e assistenciais voltadas a uma população 
alvo específica, com objetivos definidos. Embora a prevenção do óbito por câncer de mama e colo de útero guarde diferenças significativas em relação à atuação sobre fatores de risco, evolução clínica e demanda de serviços especializados, sua consideração como um conjunto se alinha às diretrizes atuais de saúde da mulher, que apontam para a integralidade do cuidado, base do funcionamento em rede dos serviços.

A definição de rede usada na análise foi a proposta pela Organização Mundial de Saúde. (OPAS, 2011, p.81): "uma rede de organizações que presta, ou faz arranjos para prestar, serviços de saúde equitativos e integrais a uma população definida e que está disposta a prestar contas por seus resultados clínicos e econômicos e pelo estado de saúde da população a que serve." As RAS (Redes de Atenção a Saúde) são constituídas por três elementos básicos: população atendida, estrutura operacional e modelo de atenção à saúde.

Nessas redes, o eixo principal é a atenção primária à população. $O$ acesso é universal e a logística de funcionamento é territorial. O planejamento e funcionamento da rede possuem quatro atributos essenciais:

a. o modelo assistencial: a atenção primária é a porta de entrada preferencial e coordena e integra a atenção à saúde, satisfazendo as necessidades da população;

b. governança e estratégia de implantação: há governança única para toda rede, que considera a equidade e os determinantes sociais;

c. organização e gestão dos serviços: os sistemas de gestão, recursos humanos, informação e logística são integrados e vinculam os membros da rede; e

d. alocação de recursos financeiros e incentivos.

O instrumento utilizado nesta pesquisa foi um questionário fechado, baseado em modelo desenvolvido pela Unicamp (LAVRAS et al, 2011) e no modelo do Programa de Melhoria do Acesso e da Qualidade da Atenção Básica (PMAQ), do Ministério da Saúde. (BRASIL, 2012a). O questionário foi construído na plataforma GoogledocsR e encaminhado, por email, aos coordenadores da Atenção Primária da região, que o enviaram aos coordenadores de unidades de atenção primária (Unidades Básicas de Saúde - UBS e Unidades de Saúde da Família - USF). O projeto foi aprovado pelo CEP/Univap, sob número 570.822, em março de 2014.

Foram incluídas, na pesquisa, todas as UBS do Litoral Norte. Os entrevistados receberam, assinaram e devolveram o Termo de Consentimento Livre e Esclarecido, ficando com uma cópia.

O questionário investigou a visão dos coordenadores sobre o funcionamento integrado, em rede, da linha de cuidado ao câncer de mama e colo de útero no município, abordando três dos quatro atributos descritos acima (a alocação de recursos não foi avaliada). Os atributos foram divididos em sete parâmetros, obtidos do modelo do PMAQ, com o qual os coordenadores estão familiarizados, mantendo 
correspondência com os atributos estabelecidos pela Organização Pan-Americana de Saúde (OPAS). (Quadro 1).

\section{Quadro 1 - Tópicos dos questionários de acordo com os atributos das OPAS}

\begin{tabular}{lc}
\hline ATRIBUTO (OPAS) & PARÂMETROS DO QUESTIONÁRIO \\
\hline Modelo Assistencial & Atenção Integral \\
Governança & Organização das ações de saúde \\
& Educação Permanente (EP) \\
& Participação e controle social \\
Organização/gestão & Gestão do trabalho \\
& Organização e integração da linha de cuidado \\
& Monitoramento e avaliação
\end{tabular}

Cada parâmetro, por sua vez, foi desmembrado em um conjunto de três a seis afirmações correspondentes ao padrão ideal para o funcionamento em rede. O sujeito da pesquisa avaliava, então, o grau de conformidade do município e de sua unidade com esse padrão, em uma escala de 1 a 5 , sendo 1 - Muito insatisfatório; 2 Insatisfatório; 3 - Regular; 4 - Satisfatório; 5 - Plenamente Satisfatório.

$\mathrm{Na}$ análise dos dados, procedeu-se uma primeira análise descritiva das características dos coordenadores e dos sete parâmetros analisados; seguida de verificação do cumprimento de cada um dos padrões que compunha os parâmetros. $O$ cumprimento dos padrões foi dicotomizado em 1 = não satisfatório, para "muito insatisfatório", "insatisfatório" e "regular"; e em 2 = satisfatório, para "satisfatório" e "plenamente satisfatório". Sempre que a frequência de respostas categorizadas como 1 foi menor do que 50\%, o padrão foi considerado não cumprido. Os dados foram analisados tendo como parâmetro a RMVale.

\section{RESULTADOS}

Foram respondidos 22 questionários no LN, correspondendo a $41 \%$ do total de unidades. Considerando que o município de São Sebastião não pode ser avaliado, pois estava em processo de reorganização administrativa e implantação de novos sistema de informação, a amostra abrangeu 59 \% das UBS dos três municípios. O LN apresentou o maior percentual de organização da atenção primária no modelo de estratégia saúde da família (100\%), em comparação às demais regiões. Em todos os tópicos do questionário, os coordenadores de Unidade de Sáude da Família (USF) do LN indicaram maior grau de conformidade aos parâmetros, em comparação à RMVale. 
No aspecto geral o município de Caraguatatuba apresentou melhores índices de avaliação, seguido de Ubatuba e Ilhabela. Os resultados obtidos nos sete tópicos dos questionários estão apresentados em forma de tabela (Tabela 2).

Considerando a frequência geral de respostas, para os coordenadores de Unidades de Atenção Primária, a rede de atendimento a pacientes com câncer de mama e colo de útero está em conformidade com os padrões estabelecidos $(62,0 \%$ de conformidade, em comparação a 54\%para a RMVale). O parâmetro de maior grau de conformidade foi "Avaliação centrada na APS" (76,4\% para o LN, 69\% para a RMVale), e dois parâmetros não atingiram conformidade ("Monitoramento e Avaliação", 43,2\% e "Participação e controle social", 33,8\%).

Três parâmetros compõem o quesito Governança. A frequência de respostas em conformidade com os padrões do parâmetro "Organização da Gestão em Nível Municipal" foi de $66,2 \%$ (em comparação a $58,2 \%$ para a RMVale) e houve conformidade com todos os padrões do parâmetro, sempre em frequência superior à RMVale (Tabela 2). O padrão mais próximo do não cumprimento diz respeito à existência de programas de educação permanente em saúde (EPS), dirigidos ao câncer feminino, que ficou no limiar do não cumprimento $(50,0 \%)$. Como cumprimento intermediário estão a existência de ações integradas; a orientação para a identificação de vulnerabilidades; a eficiência e efetividade da linha de cuidado e a tomada de decisão baseada em informação. $O$ acolhimento é o único padrão no percentil superior de conformidade (>75\%).

Já, o parâmetro "Educação Permanente em Saúde" foi considerado cumprido, porém em percentual de respostas inferior à Organização da Gestão em Nível Municipal (59,1\% para o LN; 45,7\% para a RMVale), (Tabela 2). Enquanto $63,6 \%$ dos coordenadores afirmaram ter participado de pelo menos uma atividade de educação permanente na área, apenas $54,5 \%$ disseram haver horários e locais pré-definidos para reuniões entre níveis de atenção. Nota-se coerência entre as avaliações dos padrões relativos à EPS na unidade e a avaliação da EPS promovida pela gestão, no primeiro parâmetro do questionário. A ausência de agenda e espaços de encontro entre os níveis de atenção também corrobora o desempenho baixo na integração entre os níveis de atenção, no parâmetro da articulação da linha de cuidado (42,3\% de conformidade).

O último parâmetro relativo à Governança diz respeito à gestão democrática e participativa e foi considerado em não conformidade $(33,8 \%)$, ainda que em frequência de respostas em conformidade pouco superior à RMVale (32,7\%). Esse parâmetro foi o de pior desempenho no questionário em geral. Houve acentuada não conformidade ao padrão "as unidades possuem CGU e as mulheres participam dele ativamente" ( $80 \%$ de não conformidade), e apenas $47,6 \%$ das respostas afirmaram existir canais de comunicação para as mulheres expressarem suas necessidades em saúde.

O atributo Organização e Gestão da Rede, na definição da OMS, foi avaliado por três parâmetros do questionário. Houve conformidade ao primeiro parâmetro, Organização e Integração da Linha de Cuidado (64,6\%; 54,0\% para a RMVale). Para os 
coordenadores de APS do LN, a linha de cuidado é integrada e articulada (Tabela 2); no entanto, essa percepção ancora-se, sobretudo, no acesso ao tratamento especializado (68,2\% de conformidade).

O acesso ao transporte sanitário e a presença de protocolos receberam avaliação intermediária; a estrutura da atenção primária ficou no patamar mínimo de conformidade (50,0\% das respostas) e, para os coordenadores, não há canais de comunicação estabelecidos entre os níveis de atenção $(42,9 \%$ de frequência de respostas em conformidade).

Houve conformidade ao parâmetro Gestão do Trabalho (55,6\% das respostas, Tabela 2). Porém o parâmetro é composto por dois padrões que foram avaliados de modo diferente. Enquanto a frequência de respostas em conformidade ao primeiro padrão, estabilidade do corpo profissional, foi satisfatória $(63,6 \%)$, a supervisão técnica, na área da saúde da mulher, foi considerada insatisfatória (47,6\% de conformidade). Como nas dimensões anteriores, o desempenho da rede é melhor avaliado no LN, em comparação à Região.

O último parâmetro relacionado à Organização e Gestão da Rede, Monitoramento e Avaliação não apresentou conformidade (apenas $43,2 \%$ das respostas em conformidade; na RMVale, 39,5\%). Os dados da vigilância não alcançam as unidades para fundamentar ações de vigilância e as equipes, de modo geral, não discutem os resultados do monitoramento e avaliação. Ressalte-se, no entanto, que a avaliação dos coordenadores de unidades do LN foi mais positiva do que da região.

Por fim, o Modelo Assistencial, avaliado pelo parâmetro "Atenção Integral Centrada na APS" (Tabela 2) foi o parâmetro melhor avaliado, tanto na RMVale $(69,0 \%$ de conformidade) como no LN (76,4\% de conformidade). O padrão relativo à identificação precoce de risco e busca ativa foi o melhor pontuado. Com pontuação semelhante, acima do percentil 75 , ficaram os padrões efetividade do tratamento, investigação oportuna de mulheres com exames alterados e tratamento apropriado assegurado em tempo oportuno. O item pior avaliado foi o que avalia a longitudinalidade do cuidado, embora indicado como adequado (63,6\% de conformidade). 
Tabela 2: O funcionamento em rede da linha de cuidado ao câncer ginecológico na RMVale e LN no ano de 2015.

\begin{tabular}{|c|c|c|c|c|}
\hline PARÂMETRO & $\mathbf{S}$ & PADRÕES & RMVP & LN \\
\hline \multirow{7}{*}{$\begin{array}{l}\text { Organização das } \\
\text { ações de saúde da } \\
\text { mulher em geral } \\
\text { (Nível: Gestão } \\
\text { Municipal }\end{array}$} & & A linha de cuidado é eficiente e efetiva & 64,9 & 68,2 \\
\hline & & A tomada de decisão é baseada em informação & 58,6 & 71,4 \\
\hline & & Há ações integradas de promoção da saúde & 51,3 & 63,6 \\
\hline & & Há programas de EPS dirigidas ao câncer feminino & 43,3 & 50,0 \\
\hline & & O Acolhimento é estimulado e praticado & 75,5 & 77,3 \\
\hline & & Há orientação para a identificação de vulnerabilidade & 55,7 & 66,7 \\
\hline & & PARÂMETRO & 58,2 & 66,2 \\
\hline \multirow{6}{*}{$\begin{array}{l}\text { Organização e } \\
\text { integração da linha } \\
\text { de cuidado } \\
\text { (Estrutura da rede, } \\
\text { instrumentos de } \\
\text { articulação ) }\end{array}$} & & Há protocolos instituídos para garantia de continuidade & 53,7 & 66,7 \\
\hline & & Há unidades de atenção primária e profissionais em quantidade & 43,7 & 50,0 \\
\hline & & Há canais de comunicação entre os níveis de atenção & 36,8 & 42,9 \\
\hline & & 0 acesso ao tratamento especializado é assegurado & 72,2 & 95,4 \\
\hline & & 0 acesso a transporte é garantido, se necessário & 63,5 & 68,2 \\
\hline & & PARÂMETRO & 54,0 & 64,6 \\
\hline \multirow{3}{*}{ Gestão do trabalho } & & O corpo profissional é estável (baixa rotatividade) & 59,6 & 63,6 \\
\hline & & Há supervisão técnica na área da saúde da mulher & 47,9 & 47,6 \\
\hline & & PARÂMETRO & 53,8 & 55,6 \\
\hline \multirow{3}{*}{$\begin{array}{c}\text { Participação, } \\
\text { controle social e } \\
\text { satisfação das } \\
\text { usuária } \\
\end{array}$} & & Há canais de comunicação para mulheres expressarem demandas & 44,2 & 47,6 \\
\hline & & A Unidade possui CGU e as mulheres participam dele ativamente & 21,1 & 20,0 \\
\hline & & PARÂMETRO & 32,7 & 33,8 \\
\hline \multirow{3}{*}{$\begin{array}{l}\text { Educação } \\
\text { Permanente }\end{array}$} & & Participou de pelo menos uma atividade de EPS nos últimos dois anos & 51,3 & 63,6 \\
\hline & & Há horários e locais pré-definidos para reuniões entre níveis de atenção. & 40,1 & 54,5 \\
\hline & & PARÂMETRO & 45,7 & 59,1 \\
\hline \multirow{3}{*}{$\begin{array}{l}\text { Monitoramento e } \\
\text { avaliaçã }\end{array}$} & & A unidade recebe e usa informações (SISCAN) para ações de vigilância & 44,4 & 45,4 \\
\hline & & A equipe discute os resultados do monitoramento e avaliação & 34,6 & 40,9 \\
\hline & & PARÂMETRO & 39,5 & 43,2 \\
\hline \multirow{7}{*}{$\begin{array}{l}\text { A Atenção Pimária } \\
\text { como eixo da } \\
\text { atenção integral }\end{array}$} & & A unidade realiza identificação precoce e busca ativa. & 76,2 & 86,4 \\
\hline & & Mulheres com exames alterados são investigadas em tempo oportuno & 71,9 & 77,3 \\
\hline & & 0 tratamento apropriado é assegurado em tempo oportuno & 70,7 & 72,7 \\
\hline & & O tratamento instituído é efetivo & 68,5 & 81,8 \\
\hline & & A unidade acompanha todo o processo de cuidado à mulher com câncer & 57,8 & 63,6 \\
\hline & & PARÂMETRO & 69,0 & 76,4 \\
\hline & & PONTUAÇÃO DA REDE & 54,0 & 62,0 \\
\hline
\end{tabular}

Legenda: EPS- Educação Permanente em Saúde; RMVP - Região Metropolitana do Vale do Paraíba e Litoral Norte; LN - Região de Saúde do Litoral Norte; S- Pontuação [Score] do grau de conformidade, em escala de cores.

Total de respostas: 154; Total de Respostas do LN: 22 (Município de São Sebastião não participou).

Não conformidade

$<50 \%$ respostas afirmativas
Em conformidade

50 a $<75 \%$
Conformidade com excelência

$>75 \%$ respostas afirmativas 


\section{DISCUSSÃO}

Embora a organização de um Sistema de Saúde pressuponha a organização em rede dos serviços, a diretriz de ordenamento e integração dos níveis de atenção se estabeleceu institucionalmente, no país, apenas em 2010, com a portaria ministerial 4.279 (BRASIL, 2010). A prática de avaliação dos serviços, por sua vez, recebeu reforço com o Projeto de Melhoria da Qualidade da Atenção Básica, instituído em 2012, e que tem gerado dados interessantes sobre o funcionamento da rede a partir das unidades que aderem ao Programa. Esta pesquisa avalia o momento de implantação da rede de oncologia em uma sub região de saúde paulista, composta por quatro municípios, cujo sistema se caracteriza por unidades de atenção primária, operando no modelo de estratégia de saúde da família; uma unidade de atenção secundária própria gerida por organização não governamental (Sociedade para o Desenvolvimento da Medicina, SPDM) e unidades de referência terciária em municípios de outra sub região, separados da sub-região em estudo pela barreira geográfica da Serra do Mar. A análise do questionário de avaliação, respondido por gerentes das Unidades Básicas de Saúde dos municípios de Caraguatatuba, Ilhabela e Ubatuba, pode servir como uma avaliação inicial da instituição da rede regional, e possibilitou destacar pontos fortes e fracos do funcionamento em rede da linha de cuidado ao câncer de mama e colo de útero, na região em questão. Apontamos como limitação deste estudo a não participação do Município de São Sebastião, devido ao processo de reorganização administrativa e implantação de novos sistemas de informação.

A avaliação dos coordenadores, no geral, foi positiva, embora não homogênea. $O$ modelo assistencial, com a atenção integral e organização das ações de saúde da mulher, destacou-se como ponto forte, enquanto a governança possui, simultaneamente, pontos fortes (em especial relativas a ações administrativas, de organização da rede) e fracos (em especial no que se refere à gestão participativa, relativa ao controle social e satisfação das usuárias e à política de educação permanente).

Entre os sete parâmetros investigados, o de melhor avaliação foi o funcionamento das Unidades de Saúde da Família; parâmetro de maior governabilidade dos coordenadores. O padrão mais crítico dentro desse parâmetro, no entanto, foi o acompanhamento da usuária durante todo o processo do cuidado, o que demonstra uma fragilidade do funcionamento da Estratégia de Saúde da Família. Um dos princípios da estratégia de saúde da família é, justamente, a longitudinalidade do cuidado, o apoio próximo, que vincule a mulher à unidade em todas as etapas do cuidado, desde diagnóstico ao tratamento para aumentar a efetividade do cuidado prestado. (OPAS, 2011), numa diferença entre encaminhar a paciente e prestar o cuidado integrado. Ainda que a estratégia de saúde da família tenha se ampliado no país, a sua efetivação como eixo do cuidado não tem sido verificada nos estudos que analisam o papel da APS na rede. (LAVRAS et al, 2011; FAUSTO et al, 2014) 
A articulação da rede, ou seja, a coordenação entre a APS e a atenção especializada é um dos pontos frágeis relatados na literatura. (ROSA e RADUNZ, 2013; FAUSTO et al, 2014) e que, em certa medida, foi também apontado nesta pesquisa. Embora a conformidade com os padrões do parâmetro "Integração da Linha de Cuidado" tenha alcançado $61 \%$, não há canais de comunicação estabelecidos entre a APS e os especialistas. A frequência de protocolos de encaminhamento (54,5\% para a RMVale e $66,7 \%$ no LN) é superior à encontrada por Fausto et al (2014): (52\% para câncer de colo de útero e $53 \%$ paca câncer de mama). Os autores ressaltam que os protocolos são mais frequentes nos municípios de maior porte.

Embora as respostas indiquem que o acesso ao tratamento especializado é realizado e garantido por transporte, este não se articula à rede de APS, que mantém fragilidades na estrutura física e de recursos humanos. A complementaridade desta pesquisa, que analisou a narrativa de mulheres em tratamento ou tratadas por câncer na região, revela que a existência do transporte não o redime de ser um dos pontos críticos do tratamento. Desconforto físico e emocional foram relatos frequentes, nas longas horas de espera, primeiro pelo transporte, a seguir pelo tratamento nos centros especializados; e, novamente, pelo transporte de volta ao seu município, em viagens que terminam tarde, para reiniciar na madrugada do mesmo dia. (VIANNA et al, 2015).

De modo geral, as unidades se autoavaliaram como partícipes de uma rede funcional, com a oferta de todos os recursos necessários à atenção primária às mulheres atendidas por seus serviços. É importante ressaltar que a população da região do LN é atendida, predominantemente, na rede pública de saúde (Tabela 1).

Embora tenham se auto avaliado como unidades acolhedoras, as unidades e o sistema em geral ainda não se abrem à escuta da população, ou, então, os espaços disponíveis não são apropriados pelas mulheres: a baixa participação das mulheres nos conselhos gestores é preocupante, uma vez que a participação das usuárias e sua interação com a unidade podem aprimorar o cuidado. É a partir desse elo que se avalia o funcionamento da rede, se a unidade atende às necessidades de todas as pacientes igualmente e se debatem sugestões e propostas com intuito de aprimorar os serviços prestados. Esses espaços ora não estão instituídos ora não são ativos. A despeito da obrigatoriedade constitucional e das inúmeras políticas de incentivo à participação social, bem como das conquistas dos movimentos de mulheres, observa-se que o espaço institucional das unidades de saúde para a formulação e acompanhamento de política é pouco usado nas unidades de saúde da região.

A avaliação positiva pode ser interpretada de duas maneiras. Por um lado, pode representar o gradual investimento na mudança de modelo de atenção, a adoção das políticas preconizadas pelo Ministério da Saúde. (BRASIL, 2005, 2006 e 2011); um maior investimento na rede; ou, ainda, a visão positiva dos profissionais em relação ao seu trabalho, numa valorização do próprio desempenho. De outro lado, a avaliação (como estratégia para o fortalecimento de ações ou correções de rota na gestão do 
sistema) ainda é prática pouco instituída e os coordenadores podem ter se autoconferido boas avaliações por dificuldade de autocrítica ou receio de reprimendas vindas da gestão superior, apesar da privacidade assegurada nos procedimentos de pesquisa. A institucionalização dos processos avaliativos é necessária e o Programa Nacional para Melhoria do Acesso e da Qualidade da Atenção Primária (PMAQ) pode contribuir para essa prática organizacional.

As novas diretrizes nacionais que orientam a organização das redes de cuidado, em especial para a oncologia (BRASIL, 2005), exigem governança, ou seja, demandam entendimento e capacitação do corpo técnico por parte dos gestores, clareza no modelo assistencial e organização do cuidado e da logística. Esta pesquisa mostra que a capacitação do corpo profissional, estimulada por políticas específicas para a formação no SUS (BRASIL, 2004), deve ser reforçada. A Educação é avaliada em diferentes parâmetros (Gestão do Trabalho, Educação Permanente em Saúde e Monitoramento e Avaliação) e houve concordância de respostas apontando a não conformidade a esses padrões. Sem Educação Permanente, dificilmente se instituem mudanças organizacionais. O mesmo pode ser observado em relação à incipiente prática participativa, considerada de fundamental importância para a governança. (FREY, 2004).

Estudos têm revelado que a mudança de modelo assistencial, reforçando a estratégia saúde da família como eixo do cuidado, tem ocorrido de modo gradativo, em especial para linhas de cuidado especializadas, em que a atenção secundária desempenha papel significativo no cuidado. (ONOCKO-CAMPOS, 2012).

De modo geral, a despeito da melhor situação do LN, em comparação à RMVale, se observada a série histórica de indicadores, os indicadores de saúde específicos para a avaliação do câncer de colo de útero e de mama não refletem a tendência da percepção positiva do funcionamento da rede, apresentada pelos coordenadores de unidade, pois, embora haja aumento do acesso aos serviços, a mortalidade por câncer de colo uterino é estacionária e a por câncer de mama, ascendente. Evidentemente, a relação entre a organização do cuidado e seu impacto na mortalidade não é imediata. Além de ser mediada por diversas variáveis, há que se considerar o tempo para que a melhora de um processo impacte na redução da mortalidade, em especial para doenças de longa evolução, como o câncer de colo de útero. Nesse sentido, poderia se supor que haverá redução desses indicadores no longo prazo. No entanto, é preocupante que padrões fundamentais para o aprimoramento contínuo de processos de trabalho, como o retorno da informação epidemiológica para as equipes e a educação permanente dos profissionais, com base nos indicadores, recebam avaliação regular pelos gestores locais. Nesse aspecto, poderíamos supor que a educação permanente do corpo profissional e a análise de dados epidemiológicos, fundamentando a prática, são ações aconselháveis para aprimorar os índices encontrados. 
A qualificação e organização da atenção básica não serão efetivas sem a integração aos demais níveis de atenção. A região de saúde do Litoral Norte parece estar caminhando nesse sentido e o reforço às deficiências encontradas poderá contribuir para o aprimoramento dos indicadores de processo e resultado da assistência no médio e longo prazo. A investigação acerca da integralidade da rede de cuidados e da capacitação do corpo funcional que a opera é igualmente apontada como desafio para o desenho em rede dos serviços. (FAUSTO et al, 2014). O desafio para aprimorar a linha de cuidado ao câncer de colo de útero e mama envolve a integração dos serviços, sua qualificação e abertura para a participação social; desafios que devem ser enfrentados pela gestão municipal e regional, visto que as doenças crônicas se tornarão cada vez mais presentes no âmbito urbano.

\section{CONCLUSÃO}

Neste momento de incipiente implantação da rede de oncologia, a aplicação do questionário mostrou que os coordenadores das unidades de atenção primária avaliam positivamente o funcionamento em rede das linhas de cuidado analisadas. Os coordenadores da sub-região do Litoral Norte apontaram maior conformidade aos padrões de funcionamento em rede, para a linha de cuidado do câncer de colo de útero e mama, em comparação às respostas obtidas na RMVale como um todo.

Essa percepção é coerente com o cenário epidemiológico da região de saúde do Litoral Norte, qual seja, modelo assistencial de alto percentual de unidades de atenção primária organizado segundo a estratégia saúde da família e bom desempenho nos indicadores de processos e resultados em saúde, de modo geral, quando comparada à Região Metropolitana do Vale do Paraíba.

Embora a avaliação do instrumento não tenha sido um objetivo deste estudo, ressalte-se que o questionário, uma versão simplificada do instrumento do $P M A Q$, dirigido a uma linha de cuidado específica, mostrou ser um instrumento de avaliação interessante, que poderá ser reaplicado, sistematicamente, para que se quantifique o resultado de ações voltadas ao fortalecimento da rede de cuidados.

\section{REFERÊNCIAS}

BRASIL. Ministério da Saúde. Portaria GM/MS n 198/2004, de 13 de fevereiro de 2004. Institui a política nacional de educação permanente em saúde como estratégia do Sistema Único de Saúde para a formação e o desenvolvimento de trabalhadores para o setor e dá outras providências. Disponível em: <http://www.saude.gov.br/sgtes> Acesso em: 20 ago. 2014.

BRASIL. Ministério da Saúde, Portaria 2.439. Institui a Política Nacional de Atenção Oncológica: Promoção, Prevenção, Diagnóstico, Tratamento, Reabilitação e Cuidados Paliativos, a ser implantada em todas as unidades federadas, 
respeitadas as competências das três esferas de gestão. Brasília, 2005. Disponível em: <http://www1.inca.gov.br/inca/Arquivos/Legislacao/portaria_2439.pdf>. Acesso em: 20 ago. 2014.

BRASIL, Ministério da Saúde, Secretaria de Atenção Básica, Departamento de Atenção Básica. Política Nacional da Atenção Básica. Série Pactos pela Saúde. Vol. 4. Brasilia, 2006.

BRASIL, Ministério da Saúde. Plano de Ações Estratégicas para o Enfrentamento de Doenças Crônicas Não Transmissíveis. Brasilia, 2011 Disponível em: <http://bvsms.saude.gov.br/bvs/publicacoes/plano_acoes_enfrent_dcnt_2011.pdf>. Acesso em: 20 ago. 2014.

BRASIL, DATASUS. Indicadores de Saúde. [online]. Disponível em: <http://www2.datasus.gov.br>. Acesso em 10 abr. de 2015.

BRASIL, Ministério da Saúde. Portaria MS/GM n 1.473, de 24 de junho de 2011. Institui os Comitês Gestores, Grupos Executivos, Grupos Transversais e os Comitês de Mobilização Social e de Especialistas dos compromissos prioritários de governo organizados por meio de Redes Temáticas de Atenção à Saúde. Diário Oficial da União, Brasília, DF, 24 jun. 2011.

BRASIL, Ministério da Saúde. Cadernos de Atenção Básica: Controle dos Cânceres do Colo de Útero e da Mama. Brasilia, 2013. Disponível em: <http://bvsms.saude.gov.br/bvs/publicacoes/controle_canceres_colo_utero_2013.pdf>. Acesso em: 20 ago. 2014.

BRASIL. INCA, Instituto Nacional de Câncer. A situação do câncer no Brasil. Rio de Janeiro: Instituto Nacional de Câncer, 2006-2011. Disponível em:

<http://www2.inca.gov.br>. Acesso em: 20 abr. 2015.

FAUSTO et al. A posição da Estratégia Saúde da Família na rede de atenção à saúde na perspectiva das equipes e usuários participantes do PMAQ-AB. Saúde debate. Rio de Janeiro, v. 38, n. especial, p. 13-33, 2014.

FERRERII, Ana Luiza et al. Avaliação de estratégias inovadoras na organização da Atenção Primária à Saúde. Revista Saúde Pública, v. 46, n. 1, p. 43-50, 2012. Disponível em: http://www.scielo.br/scielo.php?script=sci_arttext\&pid=S0034$89102012000100006 \&$ Ing=en\&nrm=iso>. Acesso em: 21 set. 2015.

FREY, Klaus. Artigo: Governança interativa: uma concepção para compreender a gestão pública participativa? Política \& Sociedade, v. 3, n. 5, p. 118-138, 2004.

HELBUSTO, Natália Barboza. et. al. Linha De Cuidado Ao Câncer de Colo de Útero e Mama em um Município da Região Metropolitana do Vale Do Paraíba, Segundo a Avaliação de Responsáveis por Unidades de Saúde da Família: Entre o Avanço das Diretrizes e o Desafio da Mudança. In: Encontro Latino Americano de Iniciação Científica. 18, 2014, São José Dos Campos, Anais... São José dos Campos: Universidade do Vale do Paraíba, 2014.

LAVRAS, C. Atenção primária à saúde e a organização de redes regionais de atenção à saúde no Brasil. Saúde e Sociedade, São Paulo, v. 20, n. 4, p.867-874, dez. 2011. Disponível em: <http://www.scielo.br/scielo.php?script=sci_arttext\&pid=S0104$12902011000400005 \&$ Ing=pt\&nrm=iso $>$. Acesso em: 30 nov. 2014. 
ORGANIZAÇÃO PAN-AMERICANA DA SAÚDE. A atenção à saúde coordenada pela APS: construindo as redes de atenção do SUS: contribuições para o debate. Brasília: Organização Pan-Americana da Saúde, 2011.

ROSA, Luciana Martins da e RADUNZ, Vera. Do sintoma ao tratamento adjuvante da mulher com câncer de mama. Texto \& Contexto - Enfermagem. v. 22, n.3, pp. 713721. 2013. Disponivel em <http://www.scielo.br/scielo.php?pid=S0104-

07072013000300018\&script=sci_abstract\&tlng=pt>. Acesso em: 04 jul. 2014.

VIANNA, Paula V.C. et al. Projeto de pesquisa: Observatório de Saúde da Região Metropolitana do Vale do Paraíba e Litoral Norte, SP: articulando a formação profissional e a gestão compartilhada do SUS. Relatório de Pesquisa. Processo Fapesp 2012/51787-8. Entregue a FAPESP em 31 de agosto de 2014. 